\title{
Improvements in bounded error parameter estimation using distribution theory
}

\author{
Nathalie Verdière ${ }^{1}$ and Carine Jauberthie ${ }^{2}$ and Louise Travé-Massuyès ${ }^{2}$
}

\begin{abstract}
A bounded error estimation procedure based on integro-differential polynomials linking the inputs, the outputs and the parameters of the model is presented in this paper. These polynomials are obtained from differential algebra tools given input-output polynomials. The use of the distribution theory permits to obtain new relations in which the order of derivatives of the model outputs are smaller. This method is applied on the water tank example and the results are compared with the classical method based on the simple use of inputoutput polynomials. As it will be seen, this method significantly improves the parameter estimation results.
\end{abstract}

\section{INTRODUCTION}

In this paper, an original approach to estimate parameters in the set-membership framework is presented. It is based on the use of integro-differential relations linking inputs, outputs and parameters of the model. They are obtained thanks to algebra elimination theory combined with distribution theory. From a model and an elimination order, the algebra elimination theory permits to eliminate specific variables as unknown ones in favor of inputs and outputs leading to differential polynomials called input-output polynomials ([2], [5]). The way to exploit them has resulted in several papers ([1], [3], [6], [14], [19], [22], [20]). Their use goes from identifiability analysis of the model to parameter estimation. However, in the last case, it is now well established that such relations may require to estimate high derivatives of outputs, which is an ill-posed problem. Even if some progresses have be done ([8], [7], [16], [15], [21], [18]), high derivative estimates generate important errors in inverse problems. A new approach has been recently explored based on a distribution approach [23]. It allows to obtain new relations in which the orders of derivatives are lower than those involved in the initial input-output polynomials. Indeed, multiplying these polynomials by a test function, i.e. a smooth function, and integrating permits to decrease, sometimes significantly, the order of derivatives of the model outputs. Interestingly, this method permits to annihilate part of the noise. As far as we know, this method has never been tested in the setmembership (SM) framework although it seems appropriate when complex systems are subject to unknown bounded uncertainties that make the parameter estimation task difficult ([12], [18]). Let us notice that the notion of identifiability, which is a pre-condition for safely running a parameter

\footnotetext{
${ }^{1}$ Nathalie Verdière is with Normandie Univ, UNIHAVRE, LMAH, FR-CNRS-3335, ISCN, 76600 Le Havre, France verdiern@univ-lehavre.fr

${ }^{2}$ Carine Jauberthie and Louise Travé-Massuyès are with the LAASCNRS, Université de Toulouse, CNRS, INSA, UPS, 31031 Toulouse, France cjaubert, louisedlaas. fr
}

estimation algorithm, has been extended to SM models and is now well-established [9]. SM-identifiability insures that two disjoint sets of trajectories generated by the model correspond to two disjoint value sets for the parameter vector.

The paper is organized as follows. In section II, the problem is presented and the different forms of SM-identifiability are recalled. In section III, the numerical estimation procedures based on the classical input-output polynomials and then on the integro-differential polynomials are described. They are applied and compared on the water-tank example given in section IV. Finally section V concludes the paper.

\section{PROBlem Formulation}

The models considered in this paper are uncertain nonlinear models with bounded errors, controlled or uncontrolled, of the following form:

$$
\Gamma\left\{\begin{array}{l}
\dot{x}(t, p)=f(x(t, p), u(t), p), \\
y(t, p)=h(x(t, p), p), \\
x\left(t_{0}, p\right)=x_{0} \in X_{0}, \\
p \in P \subset \mathcal{U}_{\mathcal{P}}, t_{0} \leq t \leq T,
\end{array}\right.
$$

where :

- $x(t, p) \in \mathbb{R}^{n}$ and $y(t, p) \in \mathbb{R}^{m}$ denote the state variables and the outputs at time $t$ respectively,

- $u(t) \in \mathbb{R}^{n_{u}}$ is the input vector at time $t$; in the case of uncontrolled models, $u(t)$ is equal to 0 ,

- the initial conditions $x_{0}$, if any, are assumed to belong to a bounded set $X_{0}$ and one assumes that $X_{0}$ does not contain equilibrium points of the system,

- the parameter vector $p$ belongs to a connected set $P$ assumed to be included in $\mathcal{U}_{\mathcal{P}}$, where $\mathcal{U}_{\mathcal{P}} \subseteq \mathbb{R}^{n_{p}}$ is an a priori known set of admissible parameters; the components of $p$ are denoted $p_{i}$.

- the functions $f$ and $h$ are real and analytic on $M$, where $M$ is an open set of $\mathbb{R}^{n}$ such that $x(t, p) \in M$ for every $t \in\left[t_{0}, T\right]$ and $p \in P, T$ is a finite or infinite time bound.

In the following, $Y(P)$ denotes the set of output trajectories, solution of $\Gamma$ for any $p \in P$ and is also called the output of $\Gamma$ arising from $P$.

Before establishing a parameter estimation procedure, it is recommended to perform an identifiability analysis. Indeed, an identifiable model insures that the parameter values can be uniquely inferred from output trajectories. This notion was extended in [11], [9] to the set-membership framework in which one must deal with bounded error models like $\Gamma$. Since model $\Gamma$ generates a set of trajectories, global $S M$-identifiability was proposed to compare two sets of 
trajectories, each one arising from a different connected set value for the parameter vector. The definition of global SMidentifiability states that a connected set $P^{*}$ is globally SMidentifiable if the output of $\Gamma$ arising from $P^{*}$ does not share any trajectory with the output of $\Gamma$ arising from any set $\bar{P} \subseteq P^{* c}, P^{* c}$ being the complementary of $P^{*}$ in $P$.

A stronger definition, called global $\mu$-SM-identifiable was proposed to ensure that the connected set $P^{*}$ can be contracted as small as desired while still retaining the SMidentifiability property. If the nonempty connected set $P^{*} \subseteq$ $\mathcal{U}_{\mathcal{P}}$ is globally $\mu$-SM-identifiable then it is globally SMidentifiable. However, the reciprocal is not true.

Verifying such properties on sets of trajectories is not obvious and in [9], a method based on differential relations was developed. These relations are obtained from the RosenfeldGroebner algorithm implemented in the package DifferentialAlgebra of Maple [2]. It is an elimination algorithm permitting to eliminate the unobservable variables and to obtain differential polynomials linking the inputs, the outputs and the parameters of the model.

These polynomials can be expressed as:

$$
R_{i}(y, u, p)=m_{i, 0}(y, u)+\sum_{j=1}^{q_{i}} \theta_{i, j}(p) m_{i, j}(y, u)=0
$$

with $i=1, \ldots m$ and where $\left(\theta_{i, j}(p)\right)_{1 \leq j \leq q_{i}}$ are rational in $p, \theta_{i, a} \neq \theta_{i, b}(a \neq b)$, and $\left(m_{i, j}(y, u)\right)_{1 \leq j \leq q_{i}}$ are differential polynomials with respect to $y$ and $u$ and $m_{i, 0} \neq 0$.

Let $\phi$ the real-valued function defined from the coefficients of the polynomials $R_{i}$ by :

$$
\phi: p=\left(p_{1}, \ldots, p_{p}\right) \mapsto\left(\theta_{i, j}(p)\right)_{1 \leq i \leq m, 1 \leq j \leq q_{i}} .
$$

In the case of a not bounded error model, $\phi$ is required to be injective for the model to be identifiable. In [10], [9], the authors adapted the classical injectivity definition in the framework of interval analysis to study finely global and $\mu$ SM-identifiabilities. However, in the example presented in this paper, only classical injectivity is necessary and the theorem given below has been rewritten in that sense.

To lighten the notations, we assume that $i=1$, i.e. there is only one input-output polynomial $R(y, u, p)$, and the index $i$ is omitted:

$$
R(y, u, p)=m_{0}(y, u)+\sum_{j=1}^{q} \theta_{j}(p) m_{j}(y, u)=0 .
$$

Consider $l$ the higher order derivative of $y$ in (2). $\Delta R(y, u)$ denotes the functional determinant composed of the $\left\{m_{k}(y, u)\right\}_{1 \leq k \leq q}$ and given by the Wronskian [4]

$$
\Delta R(y, u)=\left|\left(\begin{array}{ccc}
m_{1}(y, u) & \ldots & m_{q}(y, u) \\
m_{1}(y, u)^{(1)} & \ldots & m_{q}(y, u)^{(1)} \\
& \ddots & \\
m_{1}(y, u)^{(l-1)} & \ldots & m_{q}(y, u)^{(l-1)}
\end{array}\right)\right| \text {. }
$$

The following theorem links injectivity to $\mu$-SMidentifiability.
Theorem 1: Assume that the functional determinant $\Delta R(y, u)$ is not identically equal to zero ${ }^{1}$. Consider $P^{*}$ a connected subset of $\mathcal{U}_{\mathcal{P}}$. If the function $\phi$ is injective then $P^{*}$ is globally $\mu$-SM-identifiable and consequently SMidentifiable.

In the following section, the classical parameter estimation method based on the polynomial $R(y, u, p)$ is first recalled. Then, the integro-differential polynomial obtained from $R(y, u, p)$ is explained and the estimation procedure is presented.

\section{ESTIMATION PROCEDURES}

\section{A. Principles of the two methods}

We denote $y_{k}=y\left(t_{k}\right)$ the measures done at the discrete times $\left(t_{k}\right)_{1 \leq k \leq M}$, and the associated outputs $u_{k}=u\left(t_{k}\right)$. From these measures, the input-output method consists in estimating the vector composed of the so-called parameter blocks $\left(\theta_{j}(p)\right)_{j=1, \ldots, q}$ using the polynomial $R(y, u, p)$ given by (3) [22]. Under the assumption of a well-defined identifiable model, the values of the unknown parameters $p_{i}$, $i=1, \ldots, n_{p}$ can be deduced. Therefore, in what follows, system $\Gamma$ is assumed to be identifiable.

The measurement noise and uncertainties on parameters are taken into account by assuming that the output $y$ is disturbed by a bounded additive noise $\eta(t) \in[\eta(t)]$ and the parameter vector $p$ belongs to $P$ where $P$ is an interval vector. Given the polynomial $R(y, u, p)$, consider $\Theta_{j}(P)$ obtained from $\theta_{j}(p)$ by substituting $p$ by the set $P . \Theta_{j}(P)$ is a connected set for all connected sets $P$ since it only involves sum, difference and product of connected sets. For any matrix $A$, we denote $(A)_{k}$ (resp. $[A]_{k}$ ) the $k$ th line of the matrix $A$ (resp. the $k$ th line of the interval matrix $[A]$ ).

Let us now present first the classical numerical method based on the direct use of the input-output polynomials, then the method using the distribution approach, i.e. based on integro-differential polynomials.

1) Method based on input-output polynomials (refer to [18] for details): Since (3) is linear with respect to the parameter blocks $\theta_{1}(p), \ldots, \theta_{q}(p)$, if we denote the vector of parameter blocks by $\theta=$ $\left(\theta_{1}(p), \ldots, \theta_{q}(p)\right)^{T}$, the following system can be deduced

$$
A \theta=b
$$

where $(A)_{k}=\left(m_{j}\left(y_{k}, u_{k}\right)\right)_{j=1, \ldots, q}$ and $b_{k}=$ $-m_{0}\left(y_{k}, u_{k}\right)$. Considering a bounded error disturbed output, this system can be interpreted in the setmembership framework and used to estimate the unknown interval vector $\left(\Theta_{j}(P)\right)_{1 \leq j \leq q}$. It consists in

\footnotetext{
${ }^{1}$ This assumption consists in verifying the linear independence of the $m_{k}(y, u), k=1, \ldots, q$. For doing this, it is sufficient to find a time point at which the Wronskian is non-zero. In the framework of differential algebra, this condition consists in verifying that this functional determinant is not in the ideal obtained after eliminating state variables.
} 
solving the following system

$$
[A][\theta]=[b]
$$

where $[A]_{k}=\left(\left[m_{j}\left(y_{k}, u_{k}\right)\right]\right)_{j=1, \ldots, q}$ and $\left[b_{k}\right]=$ $-\left[m_{0}\left(y_{k}, u_{k}\right)\right]$. Solutions of System (6) can be found in solving the following system

$$
0 \in[A][\theta]-[b] .
$$

2) Method based on integro-differential polynomials: This method, also called the distribution approach, is based on test functions $\psi_{i}$ which are smooth functions with compact support $\operatorname{supp}\left(\psi_{i}\right)=\left[t_{i}-\epsilon, t_{i}+\epsilon\right], \epsilon>0$. More precisely, in $\mathbb{R}$, the $\psi_{i}$ 's are infinitely differentiable functions equal to zero outside a compact interval.

Assume that the component functions of $u, y$, and $x$ verify the following assumption:

(H): they are $C^{\infty}$ in $] 0, T[$ and are equal to zero on $]-\infty, 0[] T,,+\infty[$. They coincide in $] 0, T[$ with functions which are $C^{\infty}$ in $[0, T[$.

After multiplying the input-output polynomial by the test function $\psi_{i}$ and by integration by parts on $\left[t_{i}-\right.$ $\left.\epsilon, t_{i}+\epsilon\right]$, we obtain for all $i \in[1, M]$ :

$$
\begin{aligned}
& \int_{t_{i}-\epsilon}^{t_{i}+\epsilon} R(y, u, p)(s) \psi_{i}(s) d s \\
& =\int_{t_{i}-\epsilon}^{t_{i}+\epsilon} m_{1,0}(y, u)(s) \psi_{i}(s) d s \\
& +\sum_{j=1}^{q} \theta_{j}(p) \int_{t_{i}-\epsilon}^{t_{i}+\epsilon} m_{j}(y, u)(s) \psi_{i}(s) d s=0 .
\end{aligned}
$$

$M$ relations corresponding to the number of discrete times can be deduced leading to solve the following rectangular linear system:

$$
A^{\psi} \theta=b^{\psi}
$$

where $\left(A^{\psi}\right)_{i}=\left(\int_{t_{i}-\epsilon}^{t_{i}+\epsilon} m_{j}(y, u)(s) \psi_{i}(s) d s\right)_{j=1, \ldots, q}$ and $b_{i}^{\psi}=-\int_{t_{i}-\epsilon}^{t_{i}+\epsilon} m_{0}(y, u)(s) \psi_{i}(s) d s$.

As previously, the unknown interval vector of parameter blocks $\left(\Theta_{k}(P)\right)_{1 \leq k \leq q}$ is solution of

$$
\left[A^{\psi}\right][\theta]=\left[b^{\psi}\right]
$$

where $\left[A^{\psi}\right]_{i}=\left(\left[\int_{t_{i}-\epsilon}^{t_{i}+\epsilon} m_{j}(y, u)(s) \psi_{i}(s) d s\right]\right)_{j=1, \ldots, q}$ and $[b]_{i}^{\psi}=-\left[\int_{t_{i}-\epsilon}^{t_{i}+\epsilon} m_{0}(y, u)(s) \psi_{i}(s) d s\right]$. Solutions of System (10) can be obtained in solving the following system

$$
0 \in\left[A^{\psi}\right][\theta]-\left[b^{\psi}\right]
$$

\section{B. An enclosure of the initial solution}

The question that arises is: does the solution set of system $\left[A^{\psi}\right][\theta]=\left[b^{\psi}\right]$ contain the solution set of system $[A][\theta]=[b]$, otherwise what is the condition? This section answers this question by showing first, that system (9) can be seen as a perturbed system of (5) and then by giving a condition for the solution sets of systems (11) to enclose the solution sets of (7).

Let us make the following assumptions:

a) The test functions $\psi_{i}, i=1, \ldots, M$ are supposed to have the same maximal value denoted $\psi_{\max }$ and each of them reaches it at $t_{i}$,

b) for all $j=1, \ldots, q, m_{j} \psi_{i} \in C^{2}\left(\left[t_{i}-\epsilon, t_{i}+\epsilon\right]\right)$.

Let $\epsilon>0$. By the midpoint method, each coefficient $A_{i, j}^{\psi}$ and $b_{i}^{\psi}$ of matrix $A$ and vector $b$ is equal to:

$$
\begin{gathered}
\int_{t_{i}-\epsilon}^{t_{i}+\epsilon} m_{j}(s) \psi_{i}(s) d s=2 \epsilon m_{j}\left(t_{i}\right) \psi_{i}\left(t_{i}\right) \\
\left.+\frac{\epsilon^{3}}{3}\left(m_{j} \psi_{i}\right)^{\prime \prime}\left(\zeta_{i}\right), \zeta_{i} \in\right] t_{i}-\epsilon, t_{i}+\epsilon[.
\end{gathered}
$$

Let $E_{i j}=\left(m_{j} \cdot \psi_{i}\right)^{\prime \prime}\left(\zeta_{i}\right), j=1, \ldots, q$ and $F_{i}=\left(m_{0}\right.$. $\left.\psi_{i}\right)^{\prime \prime}\left(\zeta_{i}\right)$. According to Assumption (a), $\psi_{i}\left(t_{i}\right)=\psi_{\max }$ for all $i=1, \ldots, M$ and the following relations are deduced:

$\frac{A^{\psi}}{2 \epsilon}=\psi_{\max } A+\psi_{\max } \delta A, \quad \frac{b^{\psi}}{2 \epsilon}=\psi_{\max } b+\psi_{\max } \delta b$,

with

$$
\delta A=\frac{\epsilon^{2}}{6 \psi_{\max }} E, \quad \delta b=\frac{\epsilon^{2}}{6 \psi_{\max }} F,
$$

where $E=\left(E_{i, j}\right)_{\substack{i=1, \ldots, q \\ j=1, \ldots, q}}$ and $F=\left(F_{i}\right)_{i=1, \ldots, q}^{\mathrm{T}}$. Substituting equation (13) into equation (9) gives

$$
(A+\delta A) \theta=b+\delta b .
$$

Thus, system (9) is a perturbed system (5) if $\|\delta A\|_{\infty}$ and $\|\delta b\|_{\infty}$ are sufficiently small, where $\|.\|_{\infty}$ denotes the infinite induced matrix norm [17].

Let $[\gamma]$ (resp. $\left[\gamma^{\psi}\right]$ ) the solution of (7) (resp. (11)). If $[\gamma] \subseteq\left[\gamma^{\psi}\right],\left[\gamma^{\psi}\right]$ encloses the solution of (7), otherwise part of the solution can be lost. The following proposition gives a condition for ensuring that $\left[\gamma^{\psi}\right]$ encloses the solution of (7).

Proposition 3.1: $\left[\gamma^{\psi}\right]$ is the solution set of (7) if and only if $0 \in[\delta A]\left[\gamma^{\psi}\right]-[\delta b]$.

Proof: The sets of solutions of (9) and (15) being equal, $\left[\gamma^{\psi}\right]$ is the solution set of (7) if and only if 0 is contained in the connected set composed of the residual $[\delta A]\left[\gamma^{\psi}\right]-[\delta b]$.

\section{Set Inversion Via Interval Analysis}

Solving systems (7) and (11) can be done using the SIVIA algorithm (Set Inversion Via Interval Analysis) [13] for which it is necessary to give initial intervals. The problem 
that is solved here is to find $[\theta]$ such that $0 \in[A][\theta]-[b]$. We use the forward-backward propagation to contract the initial parameter box.

Consider the problem of determining a solution set $\mathbb{S}$ for the unknown quantities $\lambda$, belonging to an a priori search set $\Delta$, defined by:

$$
\mathbb{S}=\{\lambda \in \mathrm{U} \mid f(\lambda) \in[\beta]\}=f^{-1}([\beta]) \cap \Delta,
$$

where $[\beta]$ is a priori known and $f$ is a nonlinear function not necessarily invertible in the classical sense. (16) involves computing the reciprocal image of $f$ and is known as a set inversion problem which can be solved using the algorithm Set Inverter Via Interval Analysis (denoted SIVIA). The algorithm SIVIA is a recursive algorithm which explores all the search space without loosing any solution. This algorithm makes it possible to derive a guaranteed enclosure of the solution set $\mathbb{S}$ as follows:

$$
\underline{\mathbb{S}} \subseteq \mathbb{S} \subseteq \overline{\mathbb{S}}
$$

The inner enclosure $\underline{\mathbb{S}}$ is composed of the boxes that have been proved feasible. To prove that a box $[\lambda]$ is feasible, it is sufficient to prove that $f([\lambda]) \subseteq[\beta]$. Reversely, if it can be proved that $f([\lambda]) \cap[\beta]=\emptyset$, then the box $[\lambda]$ is unfeasible. Otherwise, no conclusion can be reached and the box $[\lambda]$ is said undetermined. The latter is then bisected and tested again until its size reaches a user-specified precision threshold $\varepsilon>0$. Such a termination criterion ensures that SIVIA terminates after a finite number of iterations.

Thus the algorithm SIVIA allows to obtain these two subpavings with a required precision $\varepsilon$, based on an inclusion test. The relation between the two subpavings can be characterized as:

$$
\Delta \mathbb{S}=\overline{\mathbb{S}} \backslash \underline{\mathbb{S}}
$$

where $\Delta \mathbb{S}$ is called the inclusion test uncertainty, in which no decision can be made during the test. The properties of the solution sets are:

- if $\overline{\mathbb{S}}=\emptyset$ the problem (16) has no solution,

- if $\underline{\mathbb{S}} \neq \emptyset$, there exists at least one verified solution for (16).

\section{APPLICATION}

The test functions $\psi_{i}, i=1, \ldots, M$ are bell-shaped functions centered at $t_{i}$. They are constructed from the test function: $\psi(t)=\left\{\begin{array}{l}e^{\frac{\epsilon^{2}}{t^{2}-\epsilon^{2}}} \text { if }|t|<\epsilon \\ 0 \text { if }|t| \geq \epsilon\end{array}\right.$ whose support is equal to $[-\epsilon, \epsilon]$ and for which translations and dilatations permit to define test functions on any interval. In the numerical application, we take test functions whose supports are strictly included in the time interval $[0, T]$. This assumption implies, in particular, simplifications when we use integration by parts.

In what follows, an enclosure of $\int_{a}^{b} f(s) d s$ in the setmembership framework is obtained by the interval extension of the trapezoidal classic method and it is denoted $I_{[a, b]}(f)$ in the following. The enclosures of the derivatives are obtained by using HOSM differentiators [15], [18]. The parameters of the HOSM differentiators are given by $\lambda_{0}=3, \lambda_{1}=0.2$ and $\lambda_{2}=0.1$.

\section{A. Case study}

This example concerns two coupled water tanks one above the other and modeled by:

$$
\left\{\begin{array}{l}
\dot{x}_{1}(t, p)=a_{1} u(t)-a_{2} \sqrt{x_{1}(t, p)} \\
\dot{x}_{2}(t, p)=a_{3} \sqrt{x_{1}(t, p)}-a_{4} \sqrt{x_{2}(t, p)}, \\
y_{1}(t, p)=\sqrt{x_{1}(t, p)} \\
y_{2}(t, p)=\sqrt{x_{2}(t, p)},
\end{array}\right.
$$

where $p=\left(a_{i}\right)_{i=1, \ldots, 4}, a_{i} \neq 0$, is the model parameter vector. $x=\left(x_{1}, x_{2}\right)^{T}$ represents the state vector and corresponds to the level in each tank, and $u \not \equiv 0$ is the input vector. The water level in the tanks can vary between 0 and 10.

In order to use the Rosenfeld-Groebner algorithm implemented in Maple 16, auxiliary variables $z_{1}(t, p)=\sqrt{x_{1}(t, p)}$ and $z_{2}(t, p)=\sqrt{x_{2}(t, p)}$ are introduced and the model, including the representation of the four faults, is rewritten as:

$$
\left\{\begin{array}{l}
\dot{x}_{1}(t, p)=a_{1} u(t)-a_{2} z_{1}(t, p) \\
\dot{x}_{2}(t, p)=a_{3} z_{1}(t)-a_{4} z_{2}(t, p) \\
z_{1}(t, p)^{2}=x_{1}(t, p), z_{2}(t, p)^{2}=x_{2}(t, p) \\
y_{1}(t, p)=z_{1}(t, p), y_{2}(t, p)=z_{2}(t, p)
\end{array}\right.
$$

According to the Rosenfeld-Groebner algorithm, we obtain two input-output relations:

$$
\begin{aligned}
& R_{1}(y, u, p)=-a_{1} u+a_{2} y_{1}+2 y_{1} \dot{y}_{1}, \\
& R_{2}(y, u, p)=a_{4} y_{2}-a_{3} y_{1}+2 y_{2} \dot{y}_{2}
\end{aligned}
$$

The functional determinant $\Delta R_{1}=u \dot{y}_{1}-\dot{u} y_{1}$ is not identically equal to zero provided that $u \neq 0 . \Delta R_{2}=$ $y_{2} \dot{y}_{1}-\dot{y}_{2} y_{1}$ is not identically equal to zero and the function $\phi:\left(a_{1}, a_{2}, a_{3}, a_{4}\right) \mapsto\left(a_{1}, a_{2}, a_{4}, a_{3}\right)$ is obviously injective. Thus, any connected subset $P^{*}$ is $\mu$-SM-identifiable.

\section{B. Method based on input-output polynomials}

If we denote $y_{1, p}^{(1)}\left(t_{k}\right)$ (resp. $y_{2, p}^{(1)}\left(t_{k}\right)$ ) the estimate of $\dot{y}_{1}\left(t_{k}\right)$ (resp. $\dot{y}_{2}\left(t_{k}\right)$ ), the first system which has to be solved is $\left[A_{1}\right][\theta]=\left[b_{1}\right]$ where $\left[A_{1}\right]_{k}=\left(\left[-u\left(t_{k}\right)\right],\left[y_{1}\left(t_{k}\right)\right]\right)$ and $\left[b_{1}\right]_{k}=\left[-2 y_{1}\left(t_{k}\right) y_{1, p}^{(1)}\left(t_{k}\right)\right]$.

The second system is composed of the matrix interval $\left[A_{2}\right]$ and the vector interval $\left[b_{2}\right]$ such that $\left[A_{2}\right]_{i}=$ $\left(\left[-y_{1}\left(t_{i}\right)\right],\left[y_{2}\left(t_{i}\right)\right]\right)$ and $\left[b_{2}\right]_{i}=\left[-y_{2}\left(t_{i}\right) y_{2, p}\left(t_{i}\right)\right]$.

\section{Method based on integro-differential polynomials}

For the second method, the first system to be solved is $\left[A_{1}^{\psi}\right][\theta]=\left[b_{1}^{\psi}\right]$ where

$$
\begin{aligned}
& {\left[A_{1}^{\psi}\right]_{i}=\left(\left[-I_{\left[t_{i}-\epsilon, t_{i}+\epsilon\right]}\left(u \psi_{i}\right)\right],\left[I_{\left[t_{i}-\epsilon, t_{i}+\epsilon\right]}\left(y_{1} \psi_{i}\right)\right]\right),} \\
& {\left[b_{1}^{\psi}\right]_{i}=\left[I_{\left[t_{i}-\epsilon, t_{i}+\epsilon\right]}\left(y_{1}^{2} \dot{\psi}_{i}\right)\right] .}
\end{aligned}
$$

The second one is composed of $\left[A^{\psi}\right]$ and $\left[b^{\psi}\right]$ such that:

$$
\begin{aligned}
& {\left[A_{2}^{\psi}\right]_{i}=\left(\left[-I_{\left[t_{i}-\epsilon, t_{i}+\epsilon\right]}\left(y_{1} \psi_{i}\right)\right],\left[I_{\left[t_{i}-\epsilon, t_{i}+\epsilon\right]}\left(y_{2} \psi_{i}\right)\right]\right),} \\
& {\left[b_{2}^{\psi}\right]_{i}=\left[I_{\left[t_{i}-\epsilon, t_{i}+\epsilon\right]}\left(y_{2}^{2} \dot{\psi}_{i}\right)\right] .}
\end{aligned}
$$




\section{Numerical results}

For the simulation tests, we choose $a_{1}=0.2, a_{2}=$ $0.05, a_{3}=0.1$ and $a_{4}=0.1$. The interval bounds for $\eta(t)$ are given by $[-0.10 .1]$. The initial intervals for the parameters are all given by $\mathbf{p}_{0}=\left[\begin{array}{ll}0 & 5\end{array}\right]$ and the bisection threshold for the algorithm SIVIA is 0.025 for each parameter. Figures 1 and 2 show the water level in the upper and lower tanks respectively. The estimation methods were applied on the time interval $[0,20]$ with the discrete times $\left(t_{i}\right)_{i=1, \ldots, M}$ such that the sampling period is equal to 0.1 .

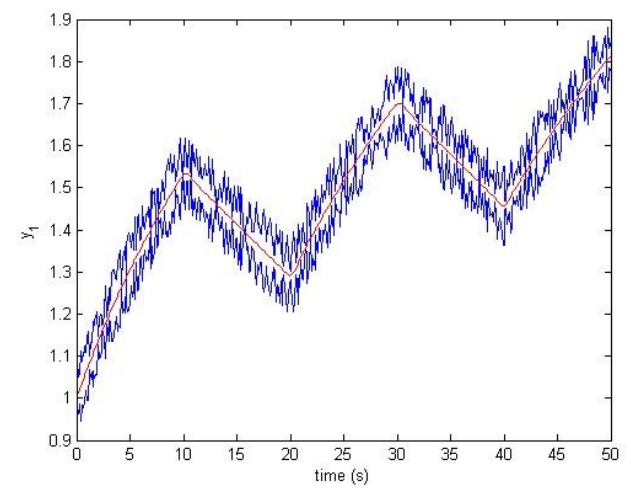

Fig. 1. Water level in the upper tank

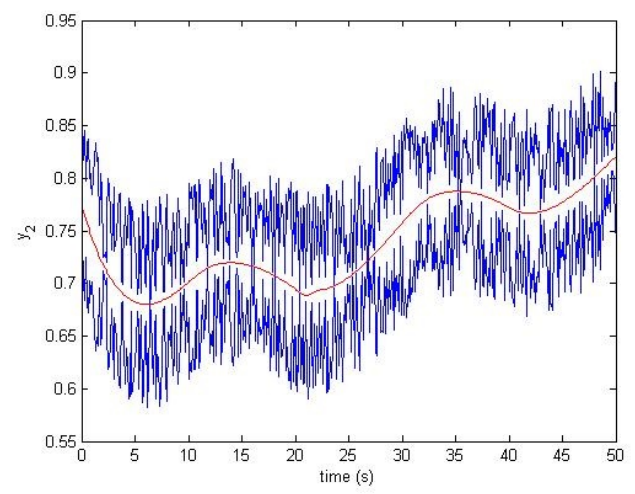

Fig. 2. Water level in the lower tank

Using the first method, the estimation results are provided by figures 3 and 4 and the computational time is 24.0036 seconds. Using the second method, we obtain figures 5 and 6 and the computational time is 16.41 seconds. In these figures, red boxes have been rejected, yellow ones (undetermined boxes) contain solutions and have a length lower than the stoping condition parameter. The green boxes (solution boxes) contain only solutions.

We see that the green boxes contain the exact values of the parameters using either the first or the second method. However, the second method provides much less conservative solutions, i.e. the green and yellow regions are much smaller.

In Tables I and II, we give the percentage of eliminated initial parameter box compared to the domains $\overline{\mathbb{S}}$ (solution

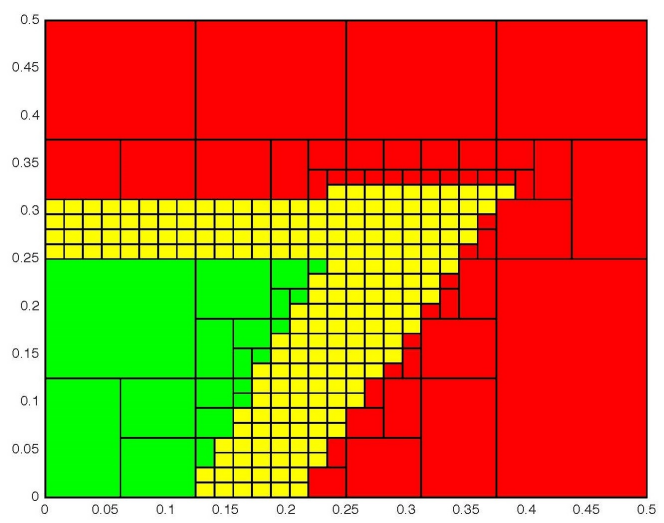

Fig. 3. Admissible parameters $a_{1}, a_{2}$ for method 1 ( $a_{1}$ on the x-axis, $a_{2}$ on the y-axis).

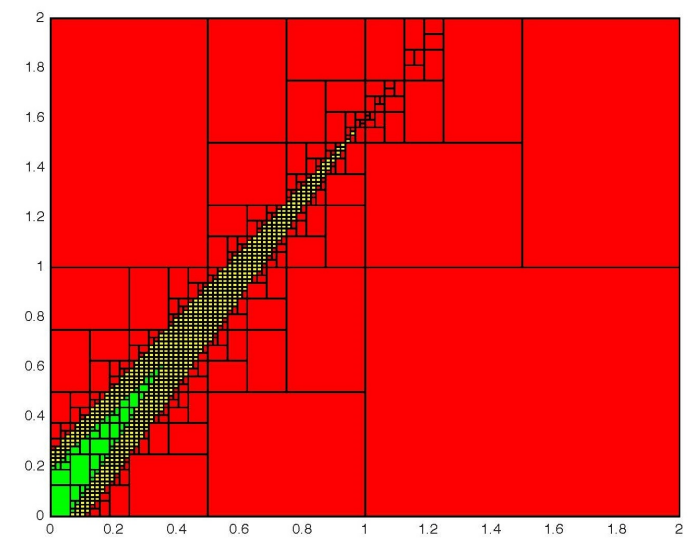

Fig. 4. Admissible parameters $a_{3}, a_{4}$ for method 1 ( $a_{3}$ on the x-axis, $a_{4}$ on the $\mathrm{y}$-axis).

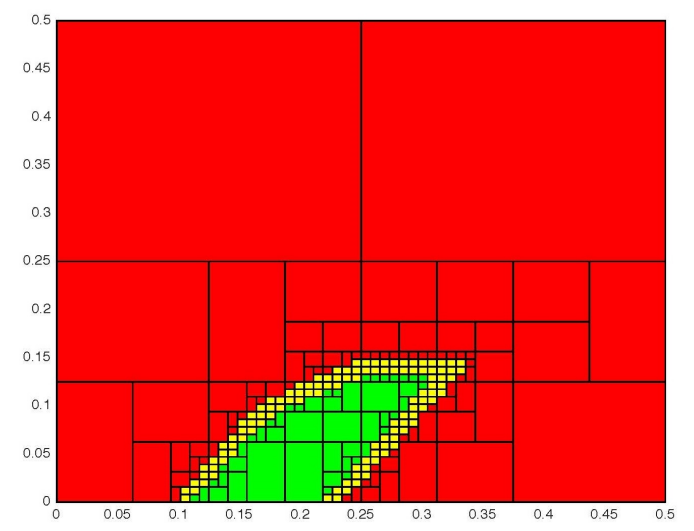

Fig. 5. Admissible parameters $a_{1}, a_{2}$ for method 2 ( $a_{1}$ on the x-axis, $a_{2}$ on the $\mathrm{y}$-axis).

and undetermined boxes) and $\underline{\mathbb{S}}$ (solution boxes only) defined in Equation (17), respectively. The eliminated percentage \% is calculated by $\% \mathbf{p}=1-\frac{w([\mathbf{p}])}{w\left(\left[\mathbf{p}_{0}\right]\right)}$ where $w([\alpha])$ means the width of the interval $[\alpha]$. 


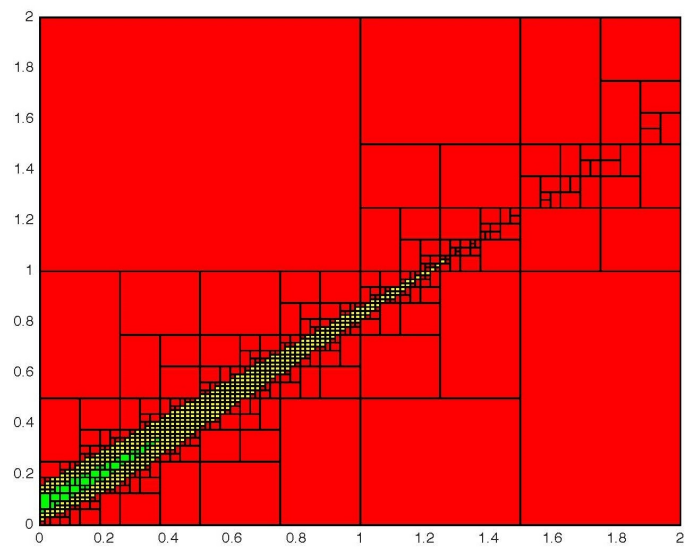

Fig. 6. Admissible parameters $a_{3}, a_{4}$ for method 2 ( $a_{3}$ on the X-axis, $a_{4}$ on the y-axis).

\begin{tabular}{ccc}
\hline Parameter & $\% \mathbf{p}_{\text {method } 1}$ & $\% \mathbf{p}_{\text {method } 2}$ \\
\hline$a_{1}$ & 86.00 & 95.00 \\
\hline$a_{2}$ & 90.00 & 97.00 \\
\hline$a_{3}$ & 82.00 & 89.00 \\
\hline$a_{4}$ & 70.00 & 91.00 \\
\hline
\end{tabular}

TABLE I

ELIMINATED PERCENTAGE OF INITIAL PARAMETER BOX FOR THE TWO METHODS (UNDETERMINED AND SOLUTION BOXES).

In both Tables I and II, we can appreciate how much the eliminated percentage of initial parameter box is higher using the second method. Interestingly, the computational time is also smaller using the second method (divided by 1.5).

\begin{tabular}{ccc}
\hline Parameter & \% $\mathbf{p}_{\text {method } 1}$ & \% $\mathbf{p}_{\text {method } 2}$ \\
\hline$a_{1}$ & 93 & 95.80 \\
\hline$a_{2}$ & 93 & 97.60 \\
\hline$a_{3}$ & 92.20 & 93.00 \\
\hline$a_{4}$ & 88.00 & 93.60 \\
\hline
\end{tabular}

TABLE II

ELIMINATED PERCENTAGE OF INITIAL PARAMETER BOX FOR THE TWO METHODS (SOLUTION BOXES).

\section{CONCLUSION}

This paper proposes an estimation method based on differential polynomials and the use of the distribution theory. It is based on integro-differential polynomials that are used to estimate the parameters of the system. This method provides very good results for the parameter estimation in the watertank problem contrary to the classical input-output polynomials based method. The explanation for the improved results is that the integro-differential polynomials contain derivatives of smaller order and integrating permits to annihilate part of the noise.

\section{REFERENCES}

[1] F. Boulier, A. Korporal, F. Lemaire, W. Perruquetti, A. Poteaux, and R. Ushirobira. An algorithm for converting nonlinear differential equations to integral equations with an application to parameter estimation from noisy data. In Proceedings of Computer Algebra in Scientific Computing 2014, Warsaw, Poland, LNCS, 8660, pages 28-43, 2014.

[2] F. Boulier, D. Lazard, F. Ollivier, and M. Petitot. Computing representation for radicals of finitely generated differential ideals. Technical report, IT-306, Université Lille I, LIFL, 59655, Villeneuve d'Ascq, 1997.

[3] F. Boulier, F. Lemaire, M. Rosenkranz, R. Ushirobira, and N. VerdiÃ̂́re. On Symbolic Approaches to Integro-Differential Equations. Chapter in Algebraic and Symbolic Computation Methods in Dynamical Systems. Springer, 2017.

[4] L. Denis-Vidal, G. Joly-Blanchard, and C. Noiret. Some effective approaches to check identifiability of uncontrolled nonlinear systems. Mathematics and Computers in Simulation, 57:35-44, 2001.

[5] L. Denis-Vidal, G. Joly-Blanchard, C. Noiret, and M. Petitot. An algorithm to test identifiability of non-linear systems. In Proceedings of 5th IFAC NOLCOS, volume 7, pages 174-178, St Petersburg, Russia, 2001.

[6] L. Denis-Vidal, G. Joly-Blanchard, and N. Verdière. Identifiability and estimation of nonlinear models: A distribution framework. In Proceedings of ECC 2007, Kos, Grèce, 2-5 juillet, 2007.

[7] M Fliess, M Mboup, H Mounier, and H Sira-Ramirez. Questioning some paradigms of signal processing via concret examples. In Proceedings of Summer School: Fast Estimation Method in Automatic Control and Signal Processing, Paris, 2005.

[8] S Ibrir and S Diop. A numerical procedure for filtering and efficient high-order signal differentiation. International Journal of Applied Mathematics and Computer Science, 14, No. 2:201-208, 2004.

[9] C. Jauberthie, L. Travé-Massuyès, and N. Verdière. Set-membership identifiability of nonlinear models and related parameter estimation properties. Journal of Applied Mathematics and Computer Science, 26(4), 2016.

[10] C. Jauberthie, N. Verdière, and L. Travé-Massuyès. Set-membership identifiability: definitions and analysis. In Proceedings of the 18th IFAC World Congress, pages 12024-12029, Milan, Italie, 2011.

[11] C. Jauberthie, N. Verdière, and L. Travé-Massuyès. Fault detection and identification relying on set-membership identifiability. Annиal Reviews in Control, 37:129-136, 2013.

[12] L. Jaulin, M. Kieffer, O. Didrit, and E. Walter. Applied Interval Analysis, with examples in parameter and state estimation, Robust control and robotics. Springer, Londres, 2001.

[13] L. Jaulin and E. Walter. Set inversion via interval analysis for nonlinear bounded-error estimation. Automatica, 29:1053-1064, 1993.

[14] G.M. Cahen J.M. Loeb. More about process identification. Automatica, pages 359-447, 1965.

[15] A. Levant. Higher-order sliding modes, differentiation and outputfeedback control. International Journal of Control, 76:924-941, 2003.

[16] M Mboup, C Join, and M Fliess. Numerical differentiation with annihilator in noisy environment. Numerical Algorithms, 50, No. 4:439-467, 2009.

[17] A. Quarteroni, R Sacco, and F Saleri. Numerical Mathematics, volume 37. Springer-Verlag Berlin Heidelberg, 2007.

[18] L. Ravanbod, C. Jauberthie, N. Verdière, and L. Travé-Massuyès. Improvements in enclosing uncertain derivatives and controlling illconditioned problems. application to interval-based fault detection and isolation. Journal of Process Control, 2017.

[19] H. Sira-Ramirez, C. Garcia Rodriguez, J. Cortes Romero, and A. Luviano Juárez. Algebraic Identification and Estimation Methods. Feedback Control Systems, Wiley, 2014.

[20] M Staroswiecki and G Comtet-Varga. Analytical redundancy relations for fault detection and isolation in algebraic dynamic systems. Automatica, 37:687-699, 2001.

[21] N. Verdière, L. Denis-Vidal, and G. Joly-Blanchard. A new method for estimating derivatives based on a distribution approach. Numerical Algorithm, 61:163 - 186, 2012.

[22] N. Verdière, L. Denis-Vidal, G. Joly-Blanchard, and D. Domurado. Identifiability and estimation of pharmacokinetic parameters for the ligands of the macrophage mannose receptor. Int. J. Appl. Math. Comput. Sci., 15:517-526, 2005.

[23] N. Verdière, S. Zhu, and L. Denis-Vidal. A distribution inputoutput polynomial approach for estimating parameters in nonlinear models. application to a chikungunya model. Accepted in Journal of Computational and Applied Mathematics, 2017. 